\title{
Efeitos do ômega-3 nas doenças inflamatórias intestinais: uma revisão da literatura
}

\author{
Effects of omega-3 fatty acids in inflammatory bowel diseases: a literature review
}

\author{
Katherine Bernart Cecconello ${ }^{1}$, Fernanda de Oliveira Marques ${ }^{2}$
}

\section{RESUMO}

Introdução: Os ácidos graxos poliinsaturados omega-3 ( $\omega-3)$ tem vários efeitos benéficos para a saúde humana. Dentre estas, possui ação antiinflamatória, diminuindo a síntese de derivados do ácido araquidônico (AA). Assim, supõe-se que a suplementação de óleo de peixe contendo ácido eicosapentaenóico (EPA) e ácido docosahexaenóico (DHA) poderia atenuar os efeitos de processos inflamatórios, diminuindo a síntese de eicosanóides precursores da inflamação, bem como o uso de medicamentos antiinflamatórios.

Objetivo: O objetivo deste artigo foi descrever os principais efeitos da terapêutica nutricional baseada na utilização da suplementação de $\omega 3$ em pacientes com doenças inflamatórias intestinais (DII) através de uma revisão da literatura.

Materiais e Métodos: Trata-se de uma revisão da literatura realizada em revistas indexadas nas bases de dados Medline, Lilacs, Scielo e Pubmed, nos idiomas português, inglês e espanhol, que abrangeu artigos publicados entre 1989 e 2014.

Resultados: De 179 referências relativas às doenças inflamatórias intestinais analisadas, após a aplicação dos critérios de inclusão e exclusão, 18 artigos foram selecionados entre estudos clínicos randomizados, observacionais, epidemiológicos, estudos de coorte e caso-controle em humanos.

Conclusão: A dose de $\omega 3$ utilizada nestes ensaios foi em média 4,5g/dia. EPA e DHA são incorporados no tecido da mucosa intestinal de pacientes com DII que complementam a alimentação com óleo de peixe e isto resulta em efeitos anti-inflamatórios com a melhora no resultado clínico, na histologia da mucosa intestinal, nos resultados da sigmoidoscopia, bem como, menor índice de recidiva e diminuição do uso de corticosteróides.

Palavras-chave: doenças inflamatórias intestinais; doença de Crohn; proctocolite; ácidos graxos ômega-3.

\begin{abstract}
Introduction: Polyunsaturated omega-3 (w3) fatty acids have many beneficial effects on human health. Among these, it has anti-inflammatory effect, decreasing the synthesis of derivatives of the arachidonic acid (AA). Therefore, it is believed that the supplementation of fish oil containing eicosapentaenoic acid (EPA) and docosahexaenoic acid (DHA) could reduce the effects of inflammatory processes by decreasing the synthesis of eicosanoid precursors of inflammation, as well as the use of anti-inflammatory drugs.

Objective: The purpose of this paper was to describe the effects of therapy based on nutritional supplementation of omega-3 in patients with inflammatory bowel disease (IBD) in a literature review.

Materials and Methods: This is a review of the literature made in journals indexed in Medline, Lilacs, Scielo and Pubmed databases, in Portuguese, English and Spanish, and covered the articles published between 1989 and 2014.

Results: Of 179 analyzed articles on inflammatory bowel disease, after inclusion and exclusion criteria were applied, 18 articles were selected from randomized clinical trials, observational, epidemiological, cohort and case-control in humans. Conclusions: The dose of $\omega 3$ used in these experiments was on average 4,5g/day. EPA and DHA are incorporated into the tissue of the intestinal mucosa of patients with IBD that complement the diet with fish oil resulting in anti-inflammatory effect with improvement in clinical outcomes, in the intestinal mucosa histology, in the results of sigmoidoscopy, as well as a lower relapse rate and decreased use of corticosteroids.
\end{abstract}

Keywords: inflammatory bowel diseases; Crohns disease; proctocolitis; omega-3 fatty acids.

\footnotetext{
${ }^{1}$ Nutricionista. Especialista em Nutrição Clínica pela Universidade de Caxias do Sul (UCS);

${ }^{2}$ Nutricionista. Professora titular da Universidade de Caxias do Sul. Mestre em Ciências Biológicas (Bioquímica) pela Universidade Federal do Rio Grande do Sul.
} 


\section{INTRODUÇÃO}

A primeira evidencia do importante papel dos ácidos graxos poliinsaturados Omega-3 ( $\omega 3$ ) na inflamação foi derivada de observações epidemiológicas da baixa incidência de doenças auto-imunes e inflamatórias como asma, psoríase e diabetes tipo 1, bem como a completa ausência de esclerose múltipla em uma população de esquimós na Groenlândia ${ }^{1}$. Descobriu-se, que o fator de proteção era promovido pelos $\omega 3$ que estão presentes em grande quantidade em alguns peixes de regiões frias, principalmente salmão, atum e truta, muito consumidos pelos esquimós ${ }^{2,3}$.

A nomenclatura ÔMEGA $(\omega)$ é definida segundo a numeração do carbono associada à primeira dupla ligação (3ํㅜ $6^{\circ}$ ou $\left.9^{\circ}\right)$, a partir do radical metila. Esta classificação implica em características estruturais e funcionais destes ácidos graxos. Existem três famílias importantes de ácidos graxos comumente consumidos na dieta: ômega $9(\omega 9)$, ômega $6(\omega 6)$ e ômega $3(\omega 3)$ sendo que apenas as duas últimas representam os ácidos graxos essenciais para o organismo. Porém, o corpo humano não os produz, sendo necessário consumi-los através dos alimentos fontes ou através de suplementos. Os lipídeos que pertencem a essas famílias: ácido alfa-linolênico (18:3 $\omega 3)$, ácido linoléico (18:2 $\omega 6)$ e ácido oléico (18:1 $\omega 9)$ usam as dessaturases e uma elongase para sintetizar seus derivados: ácido eicosapentaenóico (EPA) (20:5 w3), docosahexaenóico (DHA) $\quad(\mathrm{C} 22: 6 \quad \omega 3)$ ácido araquidônico (AA) (20:4 w6) e ácido eicosatrienóico (ETA) (20:3 w9). Em ordem de preferência, os substratos para essas enzimas são: $\omega 3>\omega 6>\omega 9$. Entretanto, existem duas classes de lipídios essenciais para a síntese dos eicosanóides: $\omega 3$ e $\omega 6$, por meio dos seus derivados EPA e $A A^{4}$.

Por meio da alimentação, pode-se obter diretamente os ácidos graxos produtores de eicosanóides. O AA pode ser obtido a partir de carnes e gorduras animais em geral, enquanto EPA e DHA são encontrados predominantemente em gordura de peixes (óleo de peixe $)^{5}$. Entre os peixes, os de origem marinha, como sardinha, salmão, arenque, truta e bacalhau, geralmente apresentam quantidades maiores de EPA/DHA do que os peixes oriundos de águas continentais. Isso ocorre, devido à expressiva quantidade desses ácidos graxos no fitoplâncton, que provê a sua distribuição ao longo da cadeia alimentar marinha ${ }^{6}$.

O $\omega 6$, está presente de forma abundante nas sementes de vegetais e nos óleos que elas produzem como o óleo de milho, açafrão, algodão, soja e girassol ${ }^{7}$. O w3, que também está presente em alguns óleos vegetais, ainda que em menor proporção que 0 ácido linoléico é encontrado em castanhas, sementes de linhaça, sementes de chia e, como citado, em óleos de peixe $^{7}$. A alimentação da população do ocidente, conhecida como dieta ocidental, é rica em ácido linoléico $(\omega 6)^{8}$. O alto consumo deste ácido graxo implica no aumento da relação $\omega 6: \omega 3$, principalmente quando a ingestão de peixe ou de óleo de peixe é baixa. Segundo Fürst $^{9}$, entre as civilizações modernas do Ocidente, essas dietas apresentam uma relação $\omega 6: \omega 3$ de 16,7:1. Esse perfil é desfavorável, especialmente nas situações em que existe uma resposta inflamatória exacerbada ${ }^{9}$

Consumo elevado de ácido linoléico favorece 0 aumento do conteúdo de AA nos fosfolipídios das membranas celulares, aumentando, consequentemente, a produção de prostaglandina E2 (PGE2) e leucotrieno B4 (LTB4), por meio das vias enzimáticas da ciclooxigenase (COX) e 5-lipoxigenase (5-LOX), respectivamente. $A$ ingestão de óleo de peixe introduz EPA nos fosfolipídios das membranas, inibindo 0 metabolismo do AA por competição pelas mesmas vias enzimáticas (COX e 5-LOX), promovendo a formação de prostaglandina E3 (PGE3), em vez de PGE2, e leucotrieno B5 (LTB5), em vez de LTB4, que são mediadores inflamatórios menos ativos ${ }^{10}$. $O$ ácido linoléico é precursor da síntese de eicosanóides da série par, com características pró-inflamatórias, como o tromboxano A2 (TXA2), PGE2 e os LTB4 ${ }^{10,11}$.

Por outro lado, o aumento da oferta de ácidos graxos da família $\omega 3$, como o ácido linolênico ou EPA e DHA, favorece a síntese de eicosanóides da série ímpar, como a PGE3, tromboxano A3 (TXA3) e LTB5, que possuem características antiinflamatórias. Esse equilíbrio proporciona menor formação de mediadores pró-inflamatórios ${ }^{12}$. O $\omega 3$ aparece nos lipídios teciduais entre um a dois dias, e tem suas concentrações 
TABELA 1 - Valores recomendados para a razão entre os ácidos graxos $\omega 6$ e $\omega 3$ na dieta.

\begin{tabular}{lll}
\hline Pais ou instituição & \multicolumn{1}{c}{$\boldsymbol{\omega 6} 6: \boldsymbol{\omega} 3$} & \multicolumn{1}{c}{ Referências } \\
\hline Canadá & $4: 1-10: 1$ & SCR $^{16}$ \\
EUA & $2: 1-3: 1$ & Simopoulos \\
EUA & $4: 1$ & Schaefer $^{18}$ \\
França & $5: 1$ & Chardigny et al. $^{19}$ \\
Japão & $2: 1-4: 1$ & Kris-Etherton et al. $^{20}$ \\
Suécia & $5: 1$ & NCM $^{21}$ \\
WHO/FAO & $5: 1-10: 1$ & WHO $^{22}$ \\
\hline
\end{tabular}

$\mathrm{WHO}=$ World Health Organization; $\mathrm{FAO}=$ Food and Agriculture Organization; $\mathrm{SRC}=$ Scientific Review Commiitte; $\mathrm{NCM}=$ Nordic Council of Ministeers.

aumentadas moderadamente nos fosfolipídios com a alimentação periódica. São depletados na maioria dos tecidos dentro de uma semana, quando a alimentação rica nesse ácido graxo é interrompida. Portanto, é necessário um consumo diário em quantidade suficiente para evitar que haja depleção do $\omega 3$ nos lipídios teciduais, a fim de se beneficiar dos efeitos que esses ácidos graxos proporcionam ${ }^{13}$.

Níveis de ingestão adequada $(\mathrm{Al})$ de ácidos graxos essenciais foram estabelecidos pelo Institute of Medicine $^{14}$, por meio das Dietary Reference Intakes (DRIs), baseadas na ingestão média da população americana. Esses valores preconizados de consumo são de $17 \mathrm{~g}$ e $12 \mathrm{~g} /$ dia de ácido linoléico $(\omega 6)$ e 1,6g e $1,1 \mathrm{~g} /$ dia de ácido linolênico ( $\omega 3$ ) para homens e mulheres, respectivamente ${ }^{14}$. Por falta de dados suficientes, o Institute of Medicine não estabeleceu Al ou DRI para AA, EPA ou $D H A^{15}$. Embora não haja DRI para EPA e DHA, tem-se recomendado que aproximadamente $10 \%$ do ácido alfa-linolênico (ALA) pode ser consumida como EPA e/ou DHA ${ }^{14}$.

Considerando que essas duas famílias de ácidos graxos competem pelas mesmas enzimas, o balanço entre $\omega 6$ e $\omega 3$ na dieta é de grande importância. Desta forma, quanto maior o consumo de $\omega 3$, menor a formação dos eicosanóides advindos da família $\omega 6$, de mais potente ação inflamatória ${ }^{7}$. De acordo com o Institute of Medicine ${ }^{14}$ a relação satisfatória da razão entre $\omega 6: \omega 3$ é de 10:1 a 5:1 e, de acordo com base em experimentação animal, esta razão é de 1:1. Atualmente, em dietas ocidentais, a relação atinge 10 a 25:1, causando um desequilíbrio dos ácidos graxos no organismo humano $^{15}$. Na tabela 1 pode-se visualizar valores de recomendação propostos por diversas organizações para a razão entre os ácidos graxos $\omega 3$ e $\omega 6$ consumidos na dieta.

Visto que o $\omega 3$ pode atenuar os efeitos de processos inflamatórios, diminuindo a síntese de eicosanóides precursores da inflamação, supõe-se que a suplementação de óleo de peixe contendo EPA e DHA altera a composição dos fosfolipídios da membrana celular e influencia na atividade das Doenças Inflamatórias Intestinais ${ }^{23}$.

Doença inflamatória intestinal (DII) é a denominação geral dada para um grupo de distúrbios inflamatórios de causa desconhecida, envolvendo o trato gastrointestinal, que pode ser dividida em dois grupos principais, a Retocolite Ulcerativa (RCU) e a Doença de Crohn (DC). DIl é uma doença idiopática, porém, existe interação entre fatores genéticos, ambientais e imunes ${ }^{24}$. A DC tem etiologia desconhecida. Caracteriza-se por uma inflamação crônica do cólon com resposta granulomatosa progressiva. Pode atingir todas áreas do trato gastrointestinal (boca até o anus). Caracterizada, também, por remissão e recaídas. As recaídas são mais prováveis de ocorrer depois que os pacientes entram em remissão ${ }^{24}$. A RCU também tem etiologia desconhecida e caracteriza-se por inflamação com ulcerações da mucosa e submucosa do intestino grosso (cólon descendente, sigmóide e reto). Estende-se de modo contínuo e possui um grande componente psicossomático. Acomete mais mulheres do que homens e é mais comum em brancos do que nas demais raças ${ }^{24}$.

O objetivo deste artigo foi descrever os principais efeitos da terapêutica nutricional baseada na utilização da suplementação de $\omega 3$ em pacientes com DII através de uma revisão da literatura.

\section{MATERIAIS E MÉTODOS}

No presente estudo realizou-se uma ampla revisão sobre o tema em artigos publicados nas revistas indexadas nas bases de dados Medline, Lilacs, Scielo e Pubmed, nos idiomas português, inglês e espanhol abrangendo artigos publicados de 1989 até 2014 . Utilizou-se os descritores: doenças inflamatórias intestinais, doença de Crohn, retocolite ulcerativa, ácidos graxos ômega-3, ácidos graxos poliinsaturados, 
EPA e DHA, nutrição e a combinação destas palavras, em inglês (inflammatory bowel diseases, Crohns disease, ulcerative colitis, omega-3 fatty acids, polyunsaturated fatty acids, EPA, DHA e nutrition). Não houve limitação na pesquisa sobre a duração da terapêutica ou a forma administrada (cápsulas, líquido, com revestimento entérico), incluindo suplementos nutricionais e fórmulas para dieta enteral.

Como critérios de inclusão foram utilizados artigos publicados nos idiomas Português, Inglês e Espanhol; artigos que continham pelo menos um dos descritores selecionados, estudos clínicos randomizados, observacionais, epidemiológicos, estudos de coorte e caso-controle em humanos. E, os critérios de exclusão: artigos em outros idiomas; estudos que não tratavam especificamente do tema, resumos, pesquisas com animais, estudos que não definiram qual foi o placebo utilizado, estudos que não mostraram desfechos de interesse e estudos que não informaram os resultados entre doença de Crohn e/ou colite ulcerativa separadamente.

Os desfechos analisados foram as taxas de remissão (para pacientes ativos) e taxa de recaída (para pacientes em remissão). Outros desfechos analisados incluíram a diminuição da atividade da doença (clínica ou endoscópica) e tempo de remissão. Estudos relatando apenas níveis de citocinas no soro/tecido, eicosanóides ou outros marcadores inflamatórios, foram excluídos. Também foram excluídos trabalhos relatando resultados agrupados em RCU e DC.

Todos os resumos encontrados foram avaliados pelos autores para ter condições de elegibilidade. Buscou-se, posteriormente, os textos completos dos artigos elegíveis. Para cada estudo selecionado, os seguintes ítens foram extraídos: (a) desenho do estudo (b) número de participantes, com referência à atividade, extensão / localização da doença, tempo de remissão (para pacientes com DII inativa), (c) detalhes sobre a intervenção terapêutica e o placebo e (d) os resultados obtidos.

Após seleção dos artigos e extração dos itens citados, realizou-se uma análise descritiva dos dados encontrados, de acordo com o tipo de doença inflamatória e se ativa ou inativa.

\section{RESULTADOS E DISCUSSÃO}

Os resultados da pesquisa de revisão da literatura estão detalhados na tabela 2.

Foram avaliadas 179 citações e resumos relacionados às DII obtidos a partir das revistas eletrônicas citadas. Dos 179 artigos encontrados, 114 foram considerados potencialmente elegíveis. Dentre estes, 10 eram resumos, 73 artigos estudaram o efeito do $\omega 3 \mathrm{em}$ animais; 4 não definiram a dosagem de $\omega 3$ e/ou placebo, 6 não relataram desfechos de interesse e 3 não diferenciaram entre DC e RCU, ou seja, informaram os resultados como DII no geral. A disposição dos estudos selecionados estão detalhados na figura 1. Após a aplicação dos critérios de inclusão e exclusão, 18 artigos $^{23,25-41}$ foram selecionados para este trabalho.

\section{Retocolite ulcerativa ativa}

Sete dos 18 artigos selecionados investigaram o efeito do $\omega 3$ em pacientes com RCU ativa ${ }^{25-30,41}$. Em três ensaios foi utilizado óleo de peixe como tratamento ativo e óleo de girassol como placebo ${ }^{27-29}$. Um destes utilizou óleo de oliva como placebo ${ }^{30}$. Outros dois trabalhos compararam os resultados entre o óleo de peixe com uma mistura de ácidos graxos ${ }^{25,26}$. Detalhes sobre o tamanho da amostra, bem como extensão e dose de $\omega 3$ utilizada estão relacionados na tabela 2 .

Um estudo em pacientes com RCU ativa informou sobre a taxa de remissão durante o período de observação $^{27}$. Quatro estudos relataram uma melhora significativa no score clínico com uso do $\omega 3$, em comparação com placebo ${ }^{25,27-29}$. Em três destes, foi acompanhada de uma melhora significativa no score endoscópico ${ }^{27-29}$ e histológico ${ }^{27}$.

Dois estudos não revelaram diferenças entre os escores clínicos e endoscópicos entre óleo de peixe ou terapia placebo ${ }^{26,30}$. No estudo de Stenson et al. ${ }^{26}$, o índice histológico melhorou significativamente durante o período de utilização de óleo de peixe.

\section{Retocolite ulcerativa Inativa}

Três estudos controlados por placebo investigaram o efeito da terapia com $\omega 3$ durante um ano ${ }^{31-33}$ e um estudo foi conduzido durante dois anos ${ }^{34}$ em pacientes 
TABELA 2 - Características dos estudos identificados sobre os efeitos do omega-3 nas doenças inflamatórias intestinais.

\begin{tabular}{|c|c|c|c|c|c|c|c|}
\hline Referência & $\begin{array}{l}\text { Delineamento } \\
\text { do estudo }\end{array}$ & Doença & $\begin{array}{c}\text { Extensão da } \\
\text { doença }\end{array}$ & $\begin{array}{c}\text { Dose de } \\
\text { EPA+DHA } \\
\text { (g/dia) ou } \\
\text { intervenção }\end{array}$ & $\begin{array}{c}\text { Duração } \\
\text { (semanas) }\end{array}$ & Placebo & Conclusões \\
\hline
\end{tabular}

\begin{abstract}
Hawthorne
et al. ${ }^{31}$,

1992

Aslan et

al. $^{25}, 1992$
\end{abstract}

ERC

RCU

ativa

Proctite
$(\mathrm{n}=4)$

CU distal

$(n=3)$

$2,7+1,8$

$\mathrm{CU}$ extensa

$(n=4)$

Stenson et
al. $^{26}, 1992$

Greenfield

et al. ${ }^{30}$,

1996

Belluzzi et

al. ${ }^{37}, 1996$

ERC

RCU

Ativa

Não informou

semanas;

após $1,1+0,75$

por 20

semanas

Ileal $(n=40)$

íleo-colônica

$(\mathrm{n}=29)$

Colônica $(n=9)$

$1,8+0,9$

52

\section{Proctite}

$(n=15)$

Loeschke et al. $^{34}, 1996$

ERDC

RCU

inativa

Distal $(n=29)$

Extensa $(\mathrm{n}=7)$

Colectomia

parcial $(n=3)$

lleal $(n=20)$

Lorenz-

Meyer et

al. $^{38}, 1996$
ERC

DC $(n=87)$

Colonica $(n=26)$

Não informada

$(n=2)$ triglicerídeos de cadeia média: $2,7 \mathrm{~g}$

de ácido

caprílico +

$1,8 \mathrm{~g}$ ácido

cáprico.

Diminuição do escore da sigmoidoscopia; diminuição da atividade da doença em RCU;

não observado efeito na atividade da doença na DC.

Diminuição da atividade da doença, diminuição do uso de corticoesteróides; não observado efeito na

sigmoidoscopia; não observado nenhum efeito sobre a histologia da mucosa intestinal.

$56 \%$ de redução da atividade da doença com óleo de peixe vs

$4 \%$ com placebo $(p<0,05)$

Diminuição do uso de corticosteróides em pacientes com recidiva; indução da remissão em pacientes com recidiva; nenhum efeito na recidiva em pacientes já em remissão. Recidiva: $42 \%$ no grupo óleo de peixe VS $48 \%$ no grupo óleo de oliva.

Cápsulas $5 \mathrm{~g}$ de ácido palmítico + 2,2g de ácido $+12,4 \mathrm{~g}$ de ácido oléico

Efeito positivo sobre a histologia da mucosa intestinal $(p=0,002)$, diminuição do uso de corticosteróide, melhora clinica com efeitos antiinflamatórios e aumento no ganho de peso.

Não observado efeito na sigmoidoscopia; nenhum efeito sobre a histologia da mucosa intestinal;

Aumento da manutenção em remissão; diminuição da recidiva. Recidiva: $28 \%$ no grupo óleo de peixe vs $69 \%$ no grupo placebo $(p<0,001)$.

Nenhum efeito sobre a histologia da mucosa intestinal; retardou a remissão, nenhum efeito sobre a recidiva.

Recidiva: $58 \%$ no grupo óleo de peixe vs $55 \%$ no grupo placebo.

Nenhum efeito sobre a recidiva. Recidiva: $57 \%$ no grupo óleo de peixe vs $55 \%$ no grupo placebo $(p<0,84)$. 
TABELA 2 - Continuação.

\begin{tabular}{|c|c|c|c|c|c|c|c|}
\hline $\begin{array}{l}\text { Mantzaris et } \\
\text { al. }^{32}, 1996\end{array}$ & ERC & $\begin{array}{l}\text { RCU } \\
\text { inativa }\end{array}$ & $\begin{array}{l}\text { Distal }(n=16) \\
\text { Extensa } \\
(n=13)\end{array}$ & $3,2+2,2$ & 52 & $\begin{array}{c}\text { 20ml/dia: } \\
\text { Óleo de oliva }\end{array}$ & $\begin{array}{c}\text { Nenhum efeito sobre a } \\
\text { recidiva ( } 27 \% \text { no grupo óleo } \\
\text { de peixe } v s 28 \% \text { no grupo } \\
\text { placebo). }\end{array}$ \\
\hline $\begin{array}{c}\text { Almallah et al. }{ }^{27}, \\
1998\end{array}$ & ERDC & $\begin{array}{l}\mathrm{RCU} \\
\text { ativa }\end{array}$ & $\begin{array}{l}\text { Proctite/ } \\
\text { Distal }\end{array}$ & $3,2+2,4$ & 24 & $\begin{array}{l}\text { Óleo de } \\
\text { girassol } \\
(2,6 \mathrm{~g} \text { de } \\
\text { ácido oléico } \\
+7,6 \mathrm{~g} \text { de } \\
\text { ácido } \\
\text { linoléico) }\end{array}$ & $\begin{array}{l}\text { Diminuição do resultado na } \\
\text { sigmoidoscopia ( } p=0,013) \text {, } \\
\text { diminuição da lesão na } \\
\text { mucosa intestinal }(p=0,016) \text {; } \\
\text { melhora da atividade clínica } \\
\text { da doença com óleo de peixe } \\
(p<0,05) \text {. }\end{array}$ \\
\hline $\begin{array}{c}\text { Almallah et al. }{ }^{29}, \\
2000\end{array}$ & ERDC & $\begin{array}{l}\text { RCU } \\
\text { Ativa }\end{array}$ & $\begin{array}{l}\text { Proctite/ } \\
\text { distal }\end{array}$ & $3,2+2,4$ & 24 & $\begin{array}{l}\text { Óleo de } \\
\text { girassol }\end{array}$ & $\begin{array}{c}\text { Diminuição na } \\
\text { sigmoidoscopia, diminuição da } \\
\text { lesão na mucosa intestinal; } \\
\text { diminuição da atividade da } \\
\text { doença. }\end{array}$ \\
\hline $\begin{array}{c}\text { Varghese and } \\
\text { Coomansingh } \\
2000\end{array}$ & ERC & $\begin{array}{l}\mathrm{RCU} \\
\text { ativa }\end{array}$ & Extensa & $\begin{array}{l}\text { Óleo de peixe: } 5,6 \mathrm{~g} \text { de } \\
\text { Omega-3 PUFA por dia }\end{array}$ & 24 & $\begin{array}{l}\text { Óleo de } \\
\text { girassol }\end{array}$ & $\begin{array}{c}\text { Diminuição da atividade da } \\
\text { doença }(p=0,001) \text {; melhora } \\
\text { dos resultados endoscópicos } \\
\qquad(p=0,054) .\end{array}$ \\
\hline $\begin{array}{l}\text { Middleton et } \\
\text { al. }^{33}, 2002\end{array}$ & ERDC & $\begin{array}{l}\text { RCU } \\
\text { inativa }\end{array}$ & $\begin{array}{c}\text { Proctite } \\
(n=25) \\
\text { Distal }(n=25) \\
\text { Extensa }(n=8)\end{array}$ & $0,27+0,05$ & 52 & $\begin{array}{l}\text { Óleo de } \\
\text { girassol }\end{array}$ & $\begin{array}{l}\text { A suplementação da dieta com } \\
\text { ácidos graxos essenciais não } \\
\text { prolongou o período de } \\
\text { remissão da doença ( } 55 \% \text { no } \\
\text { grupo óleo de peixe vs } 38 \% \text { no } \\
\text { grupo placebo } p=n . s .)\end{array}$ \\
\hline $\begin{array}{c}\text { Trebble et al. }{ }^{40} \\
2004\end{array}$ & $\mathrm{ERC}$ & $\begin{array}{c}\text { DC } \\
\text { inativa }\end{array}$ & Não informou & $2,7+2,7$ & 24 & Óleo de oliva & $\begin{array}{c}\text { O óleo de peixe foi eficaz e } \\
\text { bem tolerado; evitou recidivas } \\
\text { clínicas em pacientes com DC } \\
\text { em remissão. }\end{array}$ \\
\hline $\begin{array}{c}\text { Nielsen et al. } \\
2005\end{array}$ & ERDC & DC ativa & $\begin{array}{l}\text { lleal }(n=11) \\
\text { Íleo colônica } \\
(n=3) \\
\text { Colônica } \\
(n=17)\end{array}$ & $\begin{array}{c}\text { Fórmula ativa: } \\
\text { suplemento nutricional } \\
\text { enriquecido com } 3 \mathrm{~g} \text { de } \\
\omega 3 \text { e } 4 \mathrm{~g} \text { de } \mathrm{L}- \\
\text { arginina/dia. Fórmula } \\
\text { controle: suplemento } \\
\text { nutricional enteral com } \\
7,8 \mathrm{~g} \text { de ácido } \\
\text { linoleico/dia. }\end{array}$ & 9 & - & $\begin{array}{c}\text { Diminuição significativa na } \\
\text { atividade da doença e da } \\
\text { proteína C-reativa no grupo } \\
\text { ativo. }\end{array}$ \\
\hline $\begin{array}{c}\text { Feagan et al. } \\
2008\end{array}$ & ERC & $\begin{array}{c}\text { DC } \\
\text { inativa }\end{array}$ & Não informou & $2,0+0,8$ & 58 & $\begin{array}{l}\text { Triglicerídeo } \\
\text { s de cadeia } \\
\text { média: } \\
4 \mathrm{~g} / \mathrm{dia}\end{array}$ & $\begin{array}{c}\text { Nenhum efeito sobre a } \\
\text { recidiva; nenhum efeito sobre } \\
\text { a atividade da doença. } \\
\text { Recidiva: } 31,6 \% \text { grupo óleo de } \\
\text { peixe vs } 35,7 \% \text { com o placebo } \\
\qquad(p=0,30) .\end{array}$ \\
\hline $\begin{array}{l}\text { Uchiyama et } \\
\text { al. }^{23}, 2010\end{array}$ & ER & $\begin{array}{c}\mathrm{DC} \\
\text { inativa e } \\
\mathrm{RCU} \\
\text { inativa }\end{array}$ & Não informou & $2,7+1,8$ & 72 & - & $\begin{array}{c}\text { A intervenção com } \omega 3 \\
\text { significativamente aumentou a } \\
\text { proporção de } \omega 3 / \omega 6 \\
\text { (EPA/AA) através de uma } \\
\text { diminuição do nível de } \omega 6 \text { na } \\
\text { membrana dos eritrócitos e } \\
\text { aumento do nível de } \omega 3 \text {. Entre } \\
\text { os pacientes RCU e CD, a } \\
\text { proporção } \omega 3 / \omega 6 \text { no grupo de } \\
\text { remissão foi ignificativamente } \\
\text { mais elevados. }\end{array}$ \\
\hline $\begin{array}{c}\text { Grogan et al. } \\
2011\end{array}$ & ERDC & DC ativa & Íleo-colônica & $\begin{array}{c}\text { Fórmula ativa: dieta } \\
\text { polimérica com } 1,5 \% \\
\text { de energia provindas } \\
\text { do omega } 3 \text { e } 3 \% \text { do } \\
\text { omega } 6 \text { (ácido } \\
\text { linoléico). } \\
\text { Fórmula controle: dieta } \\
\text { elementar com } 0,4 \% \text { de } \\
\text { omega } 3 \text { e } 5,4 \% \text { de } \\
\text { omega } 6 \text {. }\end{array}$ & 6 & - & $\begin{array}{l}\text { Recidiva: } 71 \% \text { com fórmula } \\
\text { ativa vs } 70 \% \text { com fórmula } \\
\text { controle ( } p=n . s .)\end{array}$ \\
\hline
\end{tabular}

DC: doença de Crohn; RCU: retocolite ulcerativa; EPA: ácido eicosapentanóico; DHA: ácido docosahexaenóico; w3: ômega-3; w6: ômega-6; PUFA: ácidos graxos poliinsaturados; DII: doenças inflamatórias intestinais; AA: ácido aracdônico; ERC: estudo randomizado controlado por placebo; ERDC: estudo randomizado duplo-cego e controlado por placebo; ER: estudo randomizado. 
com RCU em remissão. O óleo de peixe foi utilizado em três ensaios ${ }^{31,32,34}$, enquanto que no quarto ${ }^{33}$ estudo o tratamento ativo foi uma mistura de $\omega 3$ e $\omega 6$. Azeite de oliva $^{31,32}$, óleo de milho ${ }^{34}$, e óleo de girassol $^{33}$ foram utilizados como placebos nestes ensaios. Detalhes sobre o tamanho da amostra, dose de $\omega 3$ utilizada e extensão da RCU estão resumidos na tabela 2. Os quatro $^{31-34}$ experimentos avaliaram a taxa de recaída durante o período de observação relatando uma variação entre $27 \%$ e $58 \%$ nos grupos $\omega 3$, e de $28 \%$ para $55 \%$ com o placebo ( $P$ = não significativo em todos os estudos). No estudo de Mantzaris et al. ${ }^{32}$, um benefício significativo com a utilização de óleo de peixe foi encontrado no grupo de pacientes em risco elevado de recaída (aqueles com uma história de mais de duas recidivas por ano) em 33\% no grupo óleo de peixe, em comparação com $71 \%$ no grupo placebo $(p=0,04)$.

No estudo conduzido em 2010, Uchiyama et al. ${ }^{23}$ também mostrou que a remissão, após intervenção, foi mantida em $57 \%$ dos casos e obteve uma taxa de recaída de $42,9 \%$.

\section{Doença de Crohn ativa}

Dois estudos foram identificados sobre o efeito do $\omega 3$ em DC ativa ${ }^{35,36}$. Em ambos, $\omega 3$ foi incluído na fórmula enteral para ser usado como suplemento ou nutrição enteral total. Detalhes sobre o tamanho da amostra, dose de $\omega 3$ utilizada e extensão da doença estão resumidos na tabela 2 .

No primeiro ensaio houve reduções significativas na atividade da doença e da proteína C-reativa em ambos os grupos terapêuticos ${ }^{35}$. Não foi realizada avaliação endoscópica do efeito da terapia. O segundo experimento foi conduzido em pacientes pediátricos ${ }^{36}$. O estudo comparou duas fórmulas enterais como tratamento primário para DC ativa sem nenhuma outra terapia. A taxa de remissão durante o período de observação foi relatada somente no estudo pediátrico. Não houve diferenças na taxa de remissão entre os grupos estudados $(71 \%$ com fórmula ativa e $70 \%$ com fórmula controle ${ }^{36}$ ).

\section{Doença de Crohn inativa}

$$
\text { Cinco }^{23,37,38,39,40} \text { dos } 18 \text { artigos selecionados, }
$$

investigaram o efeito do $\omega 3$ em pacientes com DC em remissão. Nestes estudos, o $\omega 3$ foi administrado para manter a remissão induzida. Quatro ${ }^{37-40}$ destes estudos foram duplo-cego, placebo-controlado. Tanto o estudo de Lorenz-Meyer et al. ${ }^{38}$, e o de Feagan et al. ${ }^{39}$ incluíram seus pacientes no estudo imediatamente após a remissão induzida por medicamentos. Ambos não conseguiram demonstrar qualquer diferença significativa na taxa de recaída entre $\omega 3$ e cápsulas de placebo.

Belluzzi et al. ${ }^{37}$, encontrou uma taxa de recaída de $28 \%$ com cápsulas de óleo de peixe e $69 \%$ com placebo $(p=0,001)$. Resultado que não pôde ser confirmado no estudo de Feagan et al. ${ }^{39}$ com taxa de recidiva de $31,6 \%$ com óleo de peixe e $35,7 \%$ com placebo.

Trebble et al. ${ }^{40}$ afirma que o óleo de peixe foi eficaz e bem tolerado. Evitou recidivas clínicas em pacientes com DC em remissão. Uchiyama et al. $^{23}$ também mostrou que a remissão após intervenção foi mantida em $79 \%$ e a taxa de recaída aconteceu em $21 \%$ dos casos.

As doses de óleo de peixe ( $\omega 3$ ) utilizadas nestes ensaios variaram entre 2,7 e 5,6 g/dia, perfazendo uma média de 4,5g/dia. As doses de EPA utilizadas foram entre 1,8 a $4,5 \mathrm{~g}$ com uma média de $2,7 \mathrm{~g}$. O DHA foi utilizado entre $0,4 \mathrm{~g}$ a $2,7 \mathrm{~g}$ e uma média de $1,6 \mathrm{~g}$. As doses propostas por estes estudos só podem ser atingidas mediante suplementação, pois a dieta, através dos alimentos fontes, não fornece tal quantidade.

Na tabela 3 estão demonstrados os benefícios do óleo de peixe encontrados nos estudos selecionados: melhora do resultado clínico, melhora na histologia da mucosa intestinal, melhora nos resultados da sigmoidoscopia, menor índice de recidiva e diminuição do uso de corticosteróides.

Segundo Lorenz et al. ${ }^{41}$ e Hawthorne et al. ${ }^{31}$, o w3 é incorporado no tecido da mucosa intestinal em pacientes com DII que suplementam a sua alimentação com óleo de peixe. Administrando oralmente, o w3 é facilmente incorporado na mucosa do intestino afetado, reduzindo assim o nível de AA e aumentando o nível de LTB5, o que induz uma resposta inflamatória fraca.

Aslan et al. ${ }^{25}$ propõe que pacientes com RCU ativa têm níveis elevados de LTB4 em sua mucosa retal, e esses níveis tendem a correlacionar com a severidade 


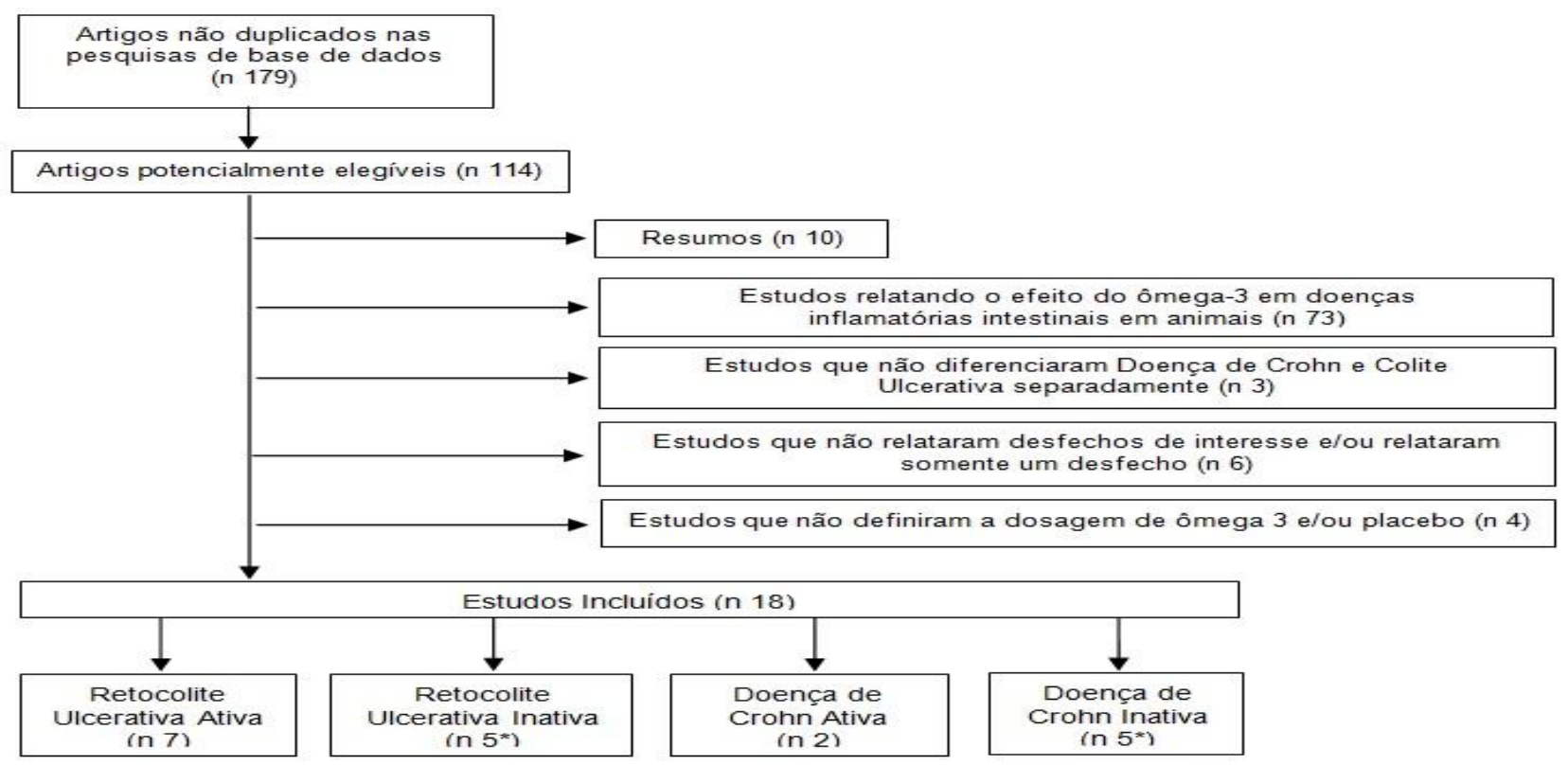

FIGURA 1 - Fluxograma da revisão. *Um artigo relatou no mesmo estudo doença de Crohn inativa e retocolite ulcerativa inativa.

da doença.

Stenson et al. ${ }^{26}$ e Hawthorne et al. ${ }^{31}$ observaram que a suplementação com óleo de peixe promove uma melhora clínica com efeitos anti-inflamatórios, tais como diminuição da produção de LTB4 produzidos pela mucosa do cólon, diminuição da produção de PGE2 e TBX2 produzidos, também, pela mucosa do cólon, e diminuição da produção de PGE2 e interferon-y. Mostraram, ainda, que a suplementação com óleo de peixe aumenta a produção do TXB5, diminui a produção do TXB4 e reduz de fato os índices de leucotrienos retais.

Almallah et al. ${ }^{27,29}$ conduziu 2 estudos, sendo um em $1998^{27}$ e outro em $2000^{29}$ com suplemento de óleo de peixe contendo 3,2g EPA + 2,4g DHA por 6 meses, mostrou que os grupos $\omega 3$, em comparação com os grupos placebo, apresentaram redução significativa na histologia, mostrada pela sigmoidoscopia. O primeiro estudo $^{29}$, estudo piloto, demonstrou que o $\omega 3$ pode diminuir a citotoxicidade natural e reduzir a atividade da doença.

Uchiyama et al. $^{23}$. sugere que as dietas e suplementos contendo ácidos graxos $\omega 3$ alteram a composição dos fosfolipídios da membrana celular e influênciam na atividade clínica das DII.

Lorenz et al. ${ }^{41}$, Almallah et al. ${ }^{27}$ e Feagan et al. ${ }^{39}$ não observaram melhora nos resultados clínicos. Aslan et al. $^{25}$, Greenfield et al. $^{30}$ e Loeschke et al. ${ }^{34}$ não observaram efeitos significativos na sigmoidoscopia e na histologia intestinal. Outros estudos ${ }^{30-34,38,39}$ também não observaram resultados significativos na recaída (tabela 3).

Os diferentes resultados relatados pelos estudos incluídos neste trabalho podem ser atribuídos às limitações da metodologia adotada, que excluiu estudos experimentais. No entanto, o objetivo desta revisão era descrever o efeito antiinflamatório da suplementação do w3 nas DII e não apenas explicar os mecanismos.

Pode-se observar, também, falta de informações sobre variáveis que são importantes para a compreensão dos efeitos do $\omega 3$ nas DII. Primeiro, porque as diferenças na dose, fonte e tipo de $\omega 3$ podem afetar os resultados clínicos, informações que poderiam ampliar o conhecimento sobre os efeitos do $\omega 3$ nestas doenças inflamatórias. Os estudos que foram revisados não relataram estas variáveis que nos permitem tirar conclusões sobre os efeitos da origem ou fonte de $\omega 3$. $E$, segundo, porque o consumo de $\omega 3$ e $\omega 6$ provindos da dieta pode influenciar os efeitos da suplementação com $\omega 3$.

Aumento do consumo de gorduras de peixe ou suplementos de óleo de peixe contendo $\omega 3$, eleva a quantidade destes ácidos graxos e seus metabólitos em células imunes humanas e, consequentemente, altera a 
TABELA 3 - Resumo dos efeitos de Omega-3 em resultados clínicos, resultados endoscópicos, resultados histológicos, recaída, remissão e uso de corticosteróides em doenças inflamatórias intestinais.

\begin{tabular}{|c|c|c|c|c|c|c|}
\hline Referência, ano & $\begin{array}{l}\text { Resultados } \\
\text { clínicos }\end{array}$ & $\begin{array}{l}\text { Resultados } \\
\text { endoscópicos }\end{array}$ & $\begin{array}{l}\text { Resultados } \\
\text { histológicos }\end{array}$ & Recaída & Remissão & $\begin{array}{l}\text { Uso de } \\
\text { corticosteróides }\end{array}$ \\
\hline Lorenz et al. ${ }^{41}, 1989$ & NRS & Melhorado & $\mathrm{NI}$ & $\mathrm{NI}$ & $\mathrm{NI}$ & $\mathrm{NI}$ \\
\hline Aslan et al. ${ }^{25}, 1992$ & Melhorado & NRS & NRS & $\mathrm{NI}$ & $\mathrm{NI}$ & Melhorado \\
\hline $\begin{array}{c}\text { Stenson et al. }{ }^{26}, \\
1992\end{array}$ & Melhorado & $\mathrm{NI}$ & Melhorado & $\mathrm{NI}$ & $\mathrm{NI}$ & Melhorado \\
\hline $\begin{array}{l}\text { Hawthorne et } \\
\text { al. }^{31}, 1992\end{array}$ & $\mathrm{NI}$ & $\mathrm{NI}$ & $\mathrm{NI}$ & NRS & NRS & Melhorado \\
\hline $\begin{array}{c}\text { Belluzzi et al. }{ }^{37}, \\
1996\end{array}$ & $\mathrm{NI}$ & $\mathrm{NI}$ & $\mathrm{NI}$ & Reduzido & $\begin{array}{l}\text { Aumento da } \\
\text { manutenção }\end{array}$ & $\mathrm{NI}$ \\
\hline $\begin{array}{c}\text { Greenfield et al. }{ }^{30}, \\
1996\end{array}$ & $\mathrm{NI}$ & $\mathrm{NI}$ & NRS & NRS & $\mathrm{NI}$ & $\mathrm{NI}$ \\
\hline $\begin{array}{c}\text { Loeschke et al. }^{34}, \\
1996\end{array}$ & Melhorado & $\mathrm{NI}$ & NRS & NRS & $\begin{array}{c}\text { Retardou } \\
\text { temporariamente }\end{array}$ & $\mathrm{NI}$ \\
\hline $\begin{array}{l}\text { Lorenz-Meyer et } \\
\quad \text { al. }^{38}, 1996\end{array}$ & $\mathrm{NI}$ & $\mathrm{NI}$ & $\mathrm{NI}$ & NRS & $\mathrm{NI}$ & $\mathrm{NI}$ \\
\hline $\begin{array}{c}\text { Mantzaris et al. }{ }^{32}, \\
1996\end{array}$ & $\mathrm{NI}$ & $\mathrm{NI}$ & $\mathrm{NI}$ & NRS & $\mathrm{NI}$ & $\mathrm{NI}$ \\
\hline $\begin{array}{c}\text { Almallah et al. }{ }^{27}, \\
1998\end{array}$ & NRS & Melhorado & Melhorado & $\mathrm{NI}$ & NRS & NRS \\
\hline $\begin{array}{c}\text { Varghese \& } \\
\text { Coomansingh }{ }^{28}, \\
2000\end{array}$ & Melhorado & Melhorado & $\mathrm{NI}$ & $\mathrm{NI}$ & $\mathrm{NI}$ & $\mathrm{NI}$ \\
\hline $\begin{array}{l}\text { Almallah et al. } .^{29} \\
2000\end{array}$ & Melhorado & $\mathrm{NI}$ & Melhorado & $\mathrm{NI}$ & $\mathrm{NI}$ & $\mathrm{NI}$ \\
\hline $\begin{array}{c}\text { Middleton et al. }{ }^{33} \text {, } \\
2002\end{array}$ & $\mathrm{NI}$ & $\mathrm{NI}$ & $\mathrm{NI}$ & NRS & $\mathrm{NI}$ & $\mathrm{NI}$ \\
\hline $\begin{array}{l}\text { Trebble et al. }{ }^{40} \\
2005\end{array}$ & Melhorado & $\mathrm{NI}$ & $\mathrm{NI}$ & Melhorado & Melhorado & $\mathrm{NI}$ \\
\hline Nielsen et al. ${ }^{35}, 2005$ & Melhorado & $\mathrm{NI}$ & $\mathrm{NI}$ & $\mathrm{NI}$ & NRS & $\mathrm{NI}$ \\
\hline $\begin{array}{l}\text { Feagan et al. } \\
2008\end{array}$ & NRS & $\mathrm{NI}$ & $\mathrm{NI}$ & NRS & $\mathrm{NI}$ & $\mathrm{NI}$ \\
\hline $\begin{array}{c}\text { Uchiyama et al. }^{23} \\
2010\end{array}$ & Melhorado & $\mathrm{NI}$ & $\mathrm{NI}$ & Melhorado & Melhorado & $\mathrm{NI}$ \\
\hline Grogan et al. ${ }^{36}, 2011$ & Melhorado & $\mathrm{NI}$ & $\mathrm{NI}$ & $\mathrm{NI}$ & Melhorado & $\mathrm{NI}$ \\
\hline
\end{tabular}

produção de importantes mediadores e reguladores da inflamação ${ }^{23}$. EPA e DHA são incorporadas no tecido da mucosa intestinal de pacientes com DII que complementam a sua dieta com óleo de peixe e de fato isto resulta em efeitos antiinflamatórios com a melhora do quadro clínico, melhora da histologia dos tecidos intestinais, melhora na pontuação da sigmoidoscopia, menor taxa de recaída, e menor uso de corticosteróides

Para conseguir estes efeitos, é proposto que utilize-se $0,1-0,2 \mathrm{~g}$ de óleo de peixe/kg/dia. Dose esta que somente será atingida mediante suplementação, pois, os alimentos fontes de $\omega 3$ não satisfazem tais doses ${ }^{42}$.
A importante influência da alimentação na formação de eicosanoides é um fato inegável, uma vez que os ácidos graxos essenciais determinam a formação de AA e EPA. A população que vive no Ocidente, incluindo os brasileiros que não vivem no litoral, tem uma ingestão muito baixa de peixes e frutos do mar. Outro fato característico da dieta do brasileiro é que o consumo de óleos vegetais tem crescido exageradamente, o que demonstra não só um baixo consumo de $\omega 3$, mas também uma elevada ingestão de $\omega 6^{43}$.

A utilização de tabelas de composição de alimentos, instrumento fundamental ao planejamento alimentar, 
representa um especial desafio para o profissional nutricionista, no sentido de definir a relação $\omega 6 / \omega 3$ na prescrição dietética, uma vez que dados relativos às concentrações específicas dos ácidos graxos presentes no alimento não se encontram facilmente. Atualmente, no Brasil, pode-se contar apenas com uma tabela de composição nutricional que apresenta o maior número de alimentos contendo estes ácidos graxos: TACO (Tabela de Composição de Alimentos da Universidade Estadual de Campinas - UNICAMP) ${ }^{44}$. Mesmo assim, não se encontram dados de alimentos das diferentes regiões brasileiras.

Segundo dados da $\operatorname{TACO}^{44}$, os peixes nacionais que apresentam uma melhor proporção entre os ácidos graxos essenciais $\omega 6$ e $\omega 3$ são bacalhau, corimba e merluza.

O nutricionista brasileiro ainda se confronta com uma dificuldade instrumental já reconhecida: a insuficiência de dados de composição de alimentos, regionais inclusive, que permita o cálculo apropriado para o planejamento dietoterápico em cada caso ${ }^{43}$. Porém, há concordância entre os autores que o benefício potencial do $\omega 3$ não deve ser negligenciado, o que indica que mais estudos clínicos nesta área devem ser realizadas a fim de que o w3 possa ser indicado como terapia auxiliar no tratamento das doenças inflamatórias intestinais. Ensaios futuros devem incluir, também, uma avaliação do total de $\omega 3$ e $\omega 6$ ingeridos na dieta, pois há falta de investigações neste tópico.

\section{REFERÊNCIAS}

1. Simopoulos AP. Omega-6/omega-3 essential fatty acid ratio and chronic diseases. Food Rev Int. 2004; 20(1):7790.

2. Simopoulos AP. n-3 fatty acids in the preventionmanagement of cardiovascular disease. Canadian Journal of Physiology and Pharmacology. 1997;75: 234-239.

3. Simopoulos AP. Omega-3 fatty acids in inflammation and autoimmune diseases. Journal of the American College of Nutrition. 2002; 21(6):495-505.

4. Bistrian BR. Clinical aspects of essential fatty acid metabolism: Jonathan Rhoads lecture. JPEN. 2003; 27(3):168.

5. Douglas CR. Patofisiologia geral: mecanismo da doença. $2^{\mathrm{a}}$ ed. São Paulo: Robe; 2000. p. 514-31.

6. Martin CA, Almeida VV, Ruiz MR, Visentainer JEL, Matshushita M, Visentainer JV. Ácidos graxos poliinsaturados ômega-3 e ômega-6: importância e ocorrência em alimentos. Rev Nutr. 2006; 19(6):761-70.

7. Mahan LK, Escott-Stump S. Lipídeos. In: Krause: alimentos, nutrição \& dietoterapia. 9ª ed. São Paulo: Roca; 1998. p. 51-3.

8. Thompson WA, Lowry SF. Effect of nutrition on inflammatory mediators. In: Zaloga GP. Nutriti on in critical care. St Louis: Mosby; 1994. p. 505-23.

9. Fürst $P$. The striking diet of the island of Crete: lipid nutrition from the palaeolithic to the affluent modern society. Clin Nutr. 2002; 21(S2):9-14.

10. James MJ, Gibson RA, Cleland LG. Dietary polyunsaturated fatty acids and inflammatory mediator production. Am J Clin Nutr. 2000; 71(S):343-8.

11. Kelley DS. Modulation of human immune and inflammatory responses by dietary fatty acids. Nutrition. 2001; 17:66973.

12. Robert O. Practical applications of fish oil (w-3 fatty acids) in primary care. J Am Board Pract. 2005; 18:28-36.

13. Frias ACD. Utilização de ácidos graxos da família ômega3 na prevenção de doenças cardiovasculares: revisão de literatura. Bauru (SP): Ed. Universidade do Sagrado Coração; 1995.

14. Institute of Medicine. Dietary Reference Intakes (DRIs) for energy, carbohydrate, fiber, fat, fatty acids, cholesterol, protein, and amino acids. Part 1. Washington (DC): National Academy Press; 2002.

15. Agency for Healthcare Research and Quality. Effects of omega-3 fatty acids on organ transplantation. Rockville (MD): Agency for Healthcare Research and Quality (US); 2005. p.115

16. Scientific Review Committee. Nutrition recommendations. Ottawa: Canadian Government Publishing Centre, Supply and Services Canada; 1990.

17. Simopoulos AP, Leaf A, Salem N. Essentiality and recommended dietary intakes for omega- 6 and omega-3 fatty acids. Ann Nutr Metabol. 1999; 43(3):127-30.

18. Schaefer EJ. Lipoproteins, nutrition, and heart disease. Am J Clin Nutr. 2002; 75(2):191-212.

19. Chardigny JM, Bretillon L, Sébédio JL. New insights in health effects of trans alpha-linolenic acid isomers in humans. Eur J Lipid Sci Technol. 2001; 103(7):478-82.

20. Kris-Etherton PM, Taylor DS, Yu-Poth S, Huth P, Moriarty $\mathrm{K}$, Fishell $\mathrm{V}$, Hargrove RL, Zhao G, Etherton TD. Polyunsaturated fatty acids in the food chain in the United States. Am J Clin Nutr. 2000; 71(1 Suppl):179S-88.

21. Nordic Council of Ministers. Nordic nutrition recommendations. Scand J Nutr. 1996; 40(4):161-5.

22. World Health Organization. Joint Consultation: fats and oils in human nutrition. Nutr Rev. 1995; 53(7):202-5.

23. Uchiyama K, Nakamura M, Odahara S, Koido S, Katahira $\mathrm{K}$, Shiraishi H, Ohkusa T, Fujise K, Tajiri H. N-3 polyunsaturated fatty acid diet therapy for patients with inflammatory bowel disease Inflamm Bowel Dis. 2010; 16(10):1696-707.

24. Silva AFM, Schieferdecker EM, Amarante HMBS. Ingestão alimentar em pacientes com doença inflamatória intestinal. Arq Bras Cir Dig. 2011;24(3):204-9.

25. Aslan A, Triadafilopoulos G. Fish oil fatty acid supplementation in active ulcerative colitis: a double-blind, placebo-controlled, crossover study. Am J Gastroenterol. 1992; 87:432-7.

26. Stenson WF, Cort D, Rodgers J, Burakoff R, DeSchyverKecskemeti K, Gramlich TL, Beeken W. Dietary supplementation with fish oil in ulcerative colitis. Ann Intern Med. 1992; 116:609-14.

27. Almallah $Y Z$, Richardson S, O'Hanrahan T, Mowat NAG, Brunt PW , Sinclair TS, Ewen S, Heys SD, Eremin O. Distal procto-colitis, natural cytotoxicity, and essential fatty acids. Am J Gastroenterol. 1998;93:804-9. 
28. Varghese TJ, Coomansingh D. Clinical response of ulcerative colitis with dietary omega-3 fatty acids: a double-blind randomized study. Br J Surg. 2000;87:73.

29. Almallah YZ, Ewen SW, El-Tahir A, Mowat NAG, Brunt, PW, Sinclair TS, Heys SD, Eremin O. Distal proctocolitis and n-3 polyunsaturated fatty acids ( $n-3$ PUFAs): The mucosal effect in situ. J Clin Immunol. 2000; 20:68-76.

30. Greenfield SM, Green AT, Teare JP, Jenkins, AP, Punchard NA, Ainley CC, Thompson RPH. A randomized controlled study of evening primrose oil and fish oil in ulcerative colitis. Aliment Pharmacol Ther. 1993; 7:159-66.

31. Hawthorne AB, Daneshmend TK, Hawkey CJ, Belluzzi A, Everitt SJ, Holmes GK, Malkinson C, Shaheen MZ, Willars JE. Treatment of ulcerative colitis with fish oil supplementation: a prospective 12 month randomised controlled trial. Gut. 1992; 33:922-8.

32. Mantzaris GJ, Archavlis E, Zografos C, Petraki K, Spiliades C, Triantafyllou G. A prospective, randomized, placebo-controlled study of fish oil in ulcerative colitis. $J$ Gastroenterol. 1996; 9:138-41.

33. Middleton SJ, Naylor S, Woolner J, Hunter JO. A doubleblind, randomized, placebo-controlled trial of essential fatty acid supplementation in the maintenance of remission of ulcerative colitis. Aliment Pharmacol Ther. 2002; 16:11315.

34. Loeschke K, Ueberschaer B, Pietsch A, Gruber E, Ewe K, Wiebecke B, Heldwein W, Lorenz R. N-3 fatty acids only delay early relapse of ulcerative colitis in remission. Dig Dis Sci. 1996;41:2087-94

35. Nielsen AA, Jorgensen LG, Nielsen JN, Eivindson $M$, Grønbaek H, Vind I, Hougaard DM, Skogstrand K, Jensen S, Munkholm P, Brandslund I, Hey H. Omega-3 fatty acids inhibit an increase of proinflammatory cytokines in patients with active Crohn's disease compared with omega- 6 fatty acids. Aliment Pharmacol Ther 2005; 22(11-12):1121-8.

36. Grogan JL, Casson DH, Terry A, Burdge GC, El-Matary W, Dalzell AM. Enteral feeding therapy for newly diagnosed pediatric Crohn's disease: A double-blind randomized controlled trial with two years follow-up Inflamm Bowel Dis. 2012; 18(2):246-53.

37. Belluzzi A, Brignola C, Campieri M, Pera A, Boschi S Miglioli M. Effect of an enteric-coated fish-oil preparation on relapses in Crohn's disease. N Engl J Med. 1996; 334:1557-60.

38. Lorenz-Meyer H, Bauer P, Nicolay C, Schulz B, Purrmann J, Fleig WE, Scheurlen C, Koop I, Pudel V, Carr L. Omega-3 fatty acids and low carbohydrate diet for maintenance of remission in Crohn's disease. A randomized controlled multicenter trial. Study Group Members (German Crohn's Disease Study Group). Scand J Gastroenterol.1996; 31:778-85.

39. Feagan BG, Sandborn WJ, Mittmann U, Bar-Meir S D'Haens G, Bradette M, Cohen A, Dallaire C, Ponich TP, McDonald JWD, Hébuterne $X$, Paré $P$, Klvana $P$, Niv $Y$, Ardizzone S, Alexeeva O, Rostom A, Kiudelis G, Spleiss J, Gilgen D, Vandervoort MK, Wong CJ, Zou GY, Donner A, Rutgeerts $P$. Omega-3 free fatty acids for the maintenance of remission in Crohn disease: The EPIC Randomized Controlled Trials. JAMA. 2008; 299:1690-7.

40. Trebble TM, Arden NK, Wootton SA, Calder PC, Mulee MA, Fine DR, Stroud MA. Fish oil and antioxidants alter the composition and function of circulating mononuclear cells in Crohn's disease. AmJ Clin Nutr. 2004; 80:1137-44.

41. Lorenz R, Weber PC, Szimnau P, Heldwein W, Strasser T, Loeschke K. Supplementation with n-3 fatty acids from fish oil in chronic inflammatory bowel disease: a randomized, placebo-controlled, double-blind cross-over trial. J InternMed Suppl. 1989;731:225-32.

42. Furst $P$, Kuhn KS. Essentiaity of recommended dietary intakes for omega 6 and omega 3 fatty acids. Ann Nut Metab, 2000; 43:127-30.

43. Gomes TKC, Oliveira SL. O papel dos ácidos graxos essenciais no perfil de eicosanoides e sua repercussão na resposta imune. Nutrire 2010; 35(1):167-86.

44. Tabela brasileira de composição de alimentos / NEPA UNICAMP. 4. ed. rev. e ampl. Campinas: NEPA UNICAMP; 2011. $161 \mathrm{p}$.

\section{Endereço para correspondência}

Katherine Bernart Cecconello

Rua Belluno $n^{\circ} 181$

Flores da Cunha/RS - CEP 95270-000

Telefone: +55 5432921181

Email: katherine.cecconello@yahoo.com.br 